\title{
Health Effects of Diseases Protected by Combined Vaccines Used in Albania
}

\author{
Eftiola Pojani $^{1}$ and Erida Nelaj ${ }^{2}$ \\ 1. Department of Pharmaceutical Sciences, Catholic University “Our Lady of Good Counsel” Tirana, Albania \\ 2. Department of Epidemiology and Infectious Disease Control, Institute of Public Health, Tirana, Albania
}

\begin{abstract}
In order to increase the convenience of application and minimize logistical problems in the recent years, the use of combined vaccines has become a growing trend. The use of combined vaccines offers benefits such as, a reduction in the number of patient visits, less complications which are associated with multiple intramuscular drilling and other risks. In 1997 the Department of Epidemiology (DE) of Institute of Public Health initiated and carried out conspicuous quantitative and qualitative modifications of up-to-them statutory notification system thus compiling the new Major Disease-Based Epidemiological Surveillance System. Mandatory reporting system on Measles/Rubella Case-Based Surveillance represents in itself an addendum of the statutory reporting system of infectious diseases. These diseases are enlisted in the Group B of the 14/Sh Form. Diphtheria is enlisted in the Group A containing the infectious diseases of the highest public health importance. They are subject of a mandatory urgent notification from the basic level. The aim of this study is to examine and check up the effectiveness of combined vaccines in our country, through the evaluation of the data from national epidemiologic surveillance verifying the distributions in time and space of these diseases in relation to the history of vaccination policies in Albania.
\end{abstract}

Key words: Vaccination program, combined vaccine, surveillance, vaccination policies.

\section{Introduction}

The use of vaccines has significantly improved global public health, preventing hospitalizations and death of millions of children every year [1]. World Health Organization (WHO) launched the Expanded Program of Immunization (EPI Expanded Program on Immunization) in 1974, and, since then, the increase in the vaccination coverage has been associated with a significant reduction in morbidity and mortality for the diseases preventable by vaccines, bringing some of them to the nearest possible limits of elimination.

The practice of vaccination is known as one of the highest efficient interferences in preventing the spread of infectious diseases. The vaccination applied on large-scale and in accordance with the appropriate strategies can lead not only to control but also the elimination of special diseases [2, 3]. In the recent

Corresponding author: Eftiola Pojani, Ph.D. student, research field: public health and immunity to infectious diseases. years a trend toward the use of combined vaccines is noticed with the purpose of increasing the practicality of application and minimizing logistical problems. During the years 1970s and 1980s, IPH (Institute of Public Health) achieved remarkable improvements in the biotechnology of the components production of $\mathrm{D}$ (diphtheria), $\mathrm{T}$ (tetanus) and $\mathrm{P}$ (pertusis) for the trivalent vaccine DTP, vaccine DT and the Td one, which together with vaccine TT, consequently led to the increasing of their effectiveness and efficiency, being reflected in a further reduction of the disease occurrence [4].

In the year 2000, Rubella component (vaccine FR) was added to Measles vaccine, and in the year 2005, another component, that of Mumps (now FRP vaccine) was added to it; and in the year 2009 Hib component (Haemophilus influenza type b) was added to then separate vaccines DTP and HepB, thus avoiding an injection to children and enabling them to receive at one time five combined vaccine antigens DTP-HepB-Hib. 
Though significant epidemiological results are achieved due to the efficacy of vaccines combinations, immunologists and microbiologists are still debating on the full equality of the immune response to the special antigens compared with those combined [5].

Despite the potential for protection against a broad spectrum of pathogens, the increasing availability of the effective vaccines can lead to a significant reduction of vaccine coverage as a result of problems related to the applicability of new vaccines according to the existing protocols. To overcome these problems, the development of combined vaccines is promoted. Their use offers benefits such as, reduction in the number of patient visits, reduced complications associated with multiple intramuscular injections, reduction in cost and administration of special vaccines, and decrease in the risk of delayed or lost vaccine. Hexavalent vaccine includes antigens against diphtheria, tetanus, acellular pertussis (DTP), hepatitis B (HBsAg), polio (P1, P2, P3) and Haemophilus influenzae type B (Hib).

Albania is making progress in the application of combined vaccines, so an effective assessment of their immune response in terms of our country would be valuable.

The objective of this paper is to investigate the effectiveness of combined vaccines in our country, through the evaluation of the data resulting from the national epidemiological surveillance for diseases such as, diphtheria, pertus, tetanus, measles, rubella, parotid and hepatitis $\mathrm{B}$ and analysis of the distributions in time and space of these diseases in relation to the history of vaccination policies in Albania. The study questions analyzed in this article are: Is the situation of infectious diseases and their complications in Albania under control after applying the selected combined vaccine? Is there a time connection between the application of these vaccines and the changes observed in infectious diseases that are prevented from them?

\section{Materials and Methods}

Epidemiological surveillance data are of fundamental importance in assessing the effectiveness of the vaccine. They are precisely those data which show during the time in progress (in years) the trend of incidence of specific infectious disease preventable-with-vaccination. The surveillance of epidemiological infectious disease is characterized by its objectives and specific criteria which enable the prevention and control of infectious disease by studying the levels of incidence and its impact on the population, epidemiological models and risk factors-companion of the disease and its potential outbreaks—and help as well, in defining scientific criteria of health policies [6].

The Albanian epidemiological surveillance system on infectious diseases has been and it continues to be a statutory one, the infectious diseases included in that system must be reported by law.

In 1997 the Department of Epidemiology (DE) of Institute of Public Health initiated and carried out conspicuous quantitative and qualitative modifications of up-to-them statutory notification system thus compiling the new Major Disease-Based Epidemiological Surveillance System (MDBSS). The new highly improved reporting system was officially approved by the Albania's Ministry of Health and put into practice starting from January 1, 1998. In the compilation of the new statutory reporting system all of the attributes of an epidemiological surveillance system were taken into account flexibility, sensitivity, acceptability. The system itself aimed thus at an active surveillance rather a passive one, increasing therefore the active case finding and laboratory capabilities for infectious diseases. The actual notification system contains 73 nosologic entities of infection diseases presented in a standard official Form (named 14/Sh). The infection diseases are divided into three groups (namely A, B, C) in that Form according to the degree of their public health importance, based on the respective measuring parameters such as the magnitude of the problem, indices of disease severity, 
socio-economic impact and preventability.

The aggregated data in the monthly 14/Sh Form are presented for each infectious diseases according to place (urban and rural), specific case definition (suspect and confirmed case), age groups. The 14/Sh monthly Form of the actual reporting system is obligatorily by law to be accompanied by the Individual Forms for each Group, which contains highly detailed epidemiological information about the case-patient thus increasing first of all the specificity of the surveillance system and quantitatively and qualitatively enriching the system epidemiological evidence.

Mandatory reporting system on Measles/Rubella Case-Based Surveillance represents in itself an addendum of the statutory reporting system of infectious diseases. These diseases are enlisted in the Group B of the 14/Sh Form and are of a rapid notification (within 1-3 days) from data sources to the local level and of a monthly notification from local level to the national one if their occurrence is represented as sporadic cases.

Diphtheria is enlisted in the Group A containing the infectious diseases of the highest public health importance. They are subject of a mandatory urgent (within $24 \mathrm{~h}$ ) notification from the basic level (data sources), through the local level (district epidemiological service) to the national one (DE of IPH) of surveillance system.

Administratively today's Albania is divided in 36 districts what does mean 36 epidemiological services and 36 microbiological laboratories. The size and the activity of the microbiological laboratories depend on the size of the respective district. The laboratory reporting system is based on regularly (monthly) mandatory notification of laboratory activity and results through the officially standard form. This system serves as a complementary one to the epidemiological surveillance system being an inherent part of the later. Based on the confirmed cases rates resulted by the monthly 14/Sh Form of the surveillance system quality level of district public health laboratories performance is thus regularly monitored [7].

The specificity versus sensitivity is a common and implict feature of a disease-based surveillnace system like ours. Based on this, it was pointed out the necessity of setting-up and implementing a complementary syndrome-beased surveillance system, thus aiming at timely detection and investigation of eventual outbreaks.

Data flow structure of the Alert System implies the weekly mandatory notification from the basic level (data sources) the national one (DE of IPH) of the surveillance system through the local level (district epidemiological service).

Paper form reporting still represents the main form of data storage at local level. Recently by the WHO support in the framework of Alert system implementation (year 2000) throughout Albania, computers are available in all district epidemiological services, what makes possible data storage parallel with the paper form storage.

Data handling includes data collection, check, aggregation and analysis in order to produce the weekly Alert Form, the monthly 14/Sh Form and yearly epidemiological report, which should be sent to the IPH.

Actually all data sources are public ones. Anyhow the private health services are by law under the mandatory notification concerning the epidemiological surveillnace of infectious diseases. The collected data in each statutory reporting system follows the same flow chart (Fig. 1).

This study is planned to be followed by another analysis that will assess the individual's immune response to combined vaccines in some Albanian population groups.

\section{Results and Discussions}

The verification of vaccine coverage of over $90 \%$ for the analized vaccines is a guarantee for the 


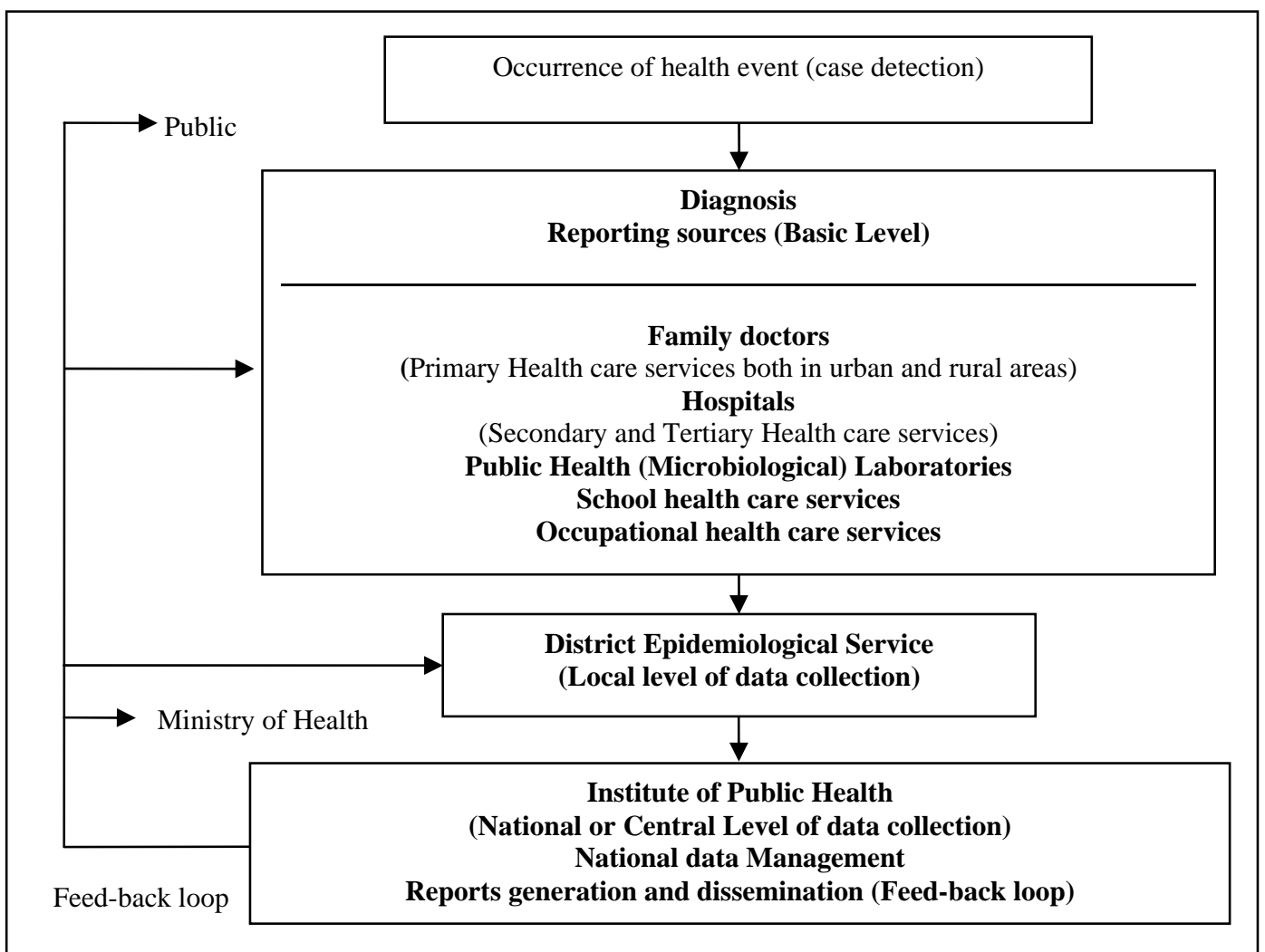

Fig. 1 System of collecting data, there are the data of epidemiological surveillance of infectious diseases, which, aswering the above questions are able to form a detailed picture of infectious diseases epidemiology in Albania.

Table 1

\begin{tabular}{lllll}
\hline \multirow{2}{*}{ Years } & \multicolumn{5}{c}{ Antigen vaccine coverage for the period 1995-2015 } \\
\cline { 2 - 4 } & $\begin{array}{l}\text { F/FR/FRP1 } \\
(\%)\end{array}$ & $\begin{array}{l}\text { DTP3 } \\
(\%)\end{array}$ & $\begin{array}{l}\text { HepB3 } \\
(\%)\end{array}$ & $\begin{array}{l}\text { DTP-HepB-Hib3 } \\
(\%)\end{array}$ \\
\hline 1995 & 90.9 & 97.0 & 88.2 & ----- \\
1996 & 91.7 & 98.1 & 96.1 & ----- \\
1997 & 91.0 & 97.5 & 95.6 & ---- \\
1998 & 89.9 & 96.6 & 94.4 & ----- \\
1999 & 83.9 & 97.6 & 91.8 & ----- \\
2000 & 91.6 & 97.5 & 97.8 & ---- \\
2001 & 95.2 & 97.7 & 98.2 & ----- \\
2002 & 96.1 & 97.8 & 95.9 & ----- \\
2003 & 95.5 & 97.3 & 97.1 & ----- \\
2004 & 96.5 & 98.2 & 98.3 & ----- \\
2005 & 97.2 & 98.4 & 98.5 & ---- \\
2006 & 92.9 & 98.2 & 97.6 & ---- \\
2007 & 95.8 & 98.4 & 97.5 & ----- \\
2008 & 98.0 & 99 & 98.7 & 97.8 \\
2009 & 97.0 & --- & ----- & 99.2 \\
2010 & 98.9 & --- & ---- & 99.1 \\
2011 & 99.0 & --- & ---- & 98.9 \\
2012 & 98.6 & --- & ---- & 98.6 \\
2013 & 98.7 & --- & ---- & 99 \\
2014 & 97.6 & --- & ---- & \\
\hline
\end{tabular}


prevention of all the other possible factors connected to the immunization program application that would influence the occurrence of the diseases, regardless of whether the antigens in the vaccine are combined or not [8]. As far as morbidity is concerned, the period 1996-2004 is characterized by very low levels of incidence: the annual total of reported cases is just 2-4 cases. In 2008 Tetanus is presented with 0 cases (in 2007 two cases) reported.

This picture is a direct indication of the effectiveness of primary prevention of tetanus and tetanus neonator through specific vaccine prophylaxis (DTP, DT, Td in pediatric age, and TT in pregnant women).

Epidemiological surveillance data on viral hepatitis (acute, unspecified), which includes four decades from 1960 to 2004, present a detailed picture of the disease occurrence as an annual frequency (number of cases) and annual incidence level (cases per 100,000 inhabitants). After a sharp decrease of the disease incidence during 1960-1962, the subsequent period of three decades 1963-1990 shows a stable trend with minor fluctuations around an average of 300 cases per 100,000 inhabitants of the annual incidence. The period 1991-2001 was characterized by a descending trend with significant fluctuations of annual incidence levels around an average value of 100 cases per 100,000 inhabitants per year. In 2008, 10 clinically suspected cases of pertus (pediatric age) were reported. It can be noticed that there is a slight decrease in the number of reported cases in comparison with the previous year (13 cases) [9]. These can be regarded as inconsiderable levels of frequency of the desease incidence among people [Table 1]. They present an overview (picture) which reflects the opltimal scale of the respective vaccine prophylaxis effectiveness. (trivalent DTP) [16-18].

A vaccination campaign, with measles and rubella components on pregnant women of childbearing age was undertaken in 2000. Since 2003 Albania has been classified as a country with no cases of measles until
2006 and 2007, when two sporadic outbreaks emerged among Roma populations. These cases were monitored and put under control after the immediate vaccination of these populations. After these cases (2006 \& 2007) there have been no more cases of measles until today [9-12].

Combined DTP-HBV-HIB vaccine versus separately administered DTP-HBV and HIB vaccines are for primary prevention of diphtheria, tetanus, pertussis, hepatitis B and Haemophilus influenzae B (HIB).

According to the results of 20 studies with 5,874 participants in an immunogenicity study (survey) it was compared the effectiveness of combined DTP-HBV-HIB vaccines versus combined DTP-HBV and separate HIB vaccinations. [19]. Minor adverse events such as pain and redness were more common in children given the combined vaccine. Overall, the direction shown by the results is in favour of the DTPw (diptheria-tetanus-whole cell pertussis)-HBV-HIB vaccine rather than the DTPa (diphtheria-tetanus-acellular pertussis)-HBV-HIB vaccine when compared to the separate vaccines.

But, what are the immunological profiles of the population against infectious disease preventable-with-vaccination? In other words, what are the general and specific levels of age-specific population protection against that disease?

The answers to these questions is seroepidemiology. Cross-sectional seroepidemiology surveys represent a valuable complementary epidemiological surveillance data in evaluating the effectiveness of the vaccine (vaccination program) [13, 14]. Although specific protection serum levels are obtained by cross-sectional seroepidemiology surveys, they provide a clear picture, which, in essence, is nothing but evaluation of the vaccine effectiveness.

Furthermore, the performance about the specific post vaccination protection levels in years is obtained via cross-sectional seroepidemiologic surveys. They are described as the only accurate instrument in 
determining the eventual need of dose/booster doses (Re-Vaccination/revaccination) in the vaccination program. The postulate that the vaccine can not give specific absolute protection is well-known: its efficiency levels can reach up to $98-99 \%$ at the best case. It is also known that the vaccine effectiveness is always displayed in lower levels than its efficiency level.

The direct and indirect post vaccination protection represent, in essence, the so-called "herd immunity", which is, after all, connected with elimination/eradication of infectious disease prevented by vaccination.

The World Health Organization has recently estimated the global levels of vaccine coverage (2012).

Actually, immunization prevents two to three million deaths a year caused by diphtheria, tetanus, pertussis and measles.

During the evaluation of vaccination with three doses of DTP vaccine an increase in coverage from $20 \%$ in 1980 and $70 \%$ in 2000 to $83 \%$ in 2012 was observed.

In our country, as mentioned above, HepB vaccination started in 1994 but without a detailed prior plan [14, 15]. During 2000-2003 the vaccination program undertook a massive campaign with the scope of vaccinating healthcare personnel. In 2010-2012 another vaccination campaign was initiated. It included all children who were born in 1992-1993 and the rest of the unvaccinated children, born in the first months of 1994, when vaccination with hepatitis B started.

\section{Conclusions}

The use of combined vaccines offers benefits such as: reduction in the number of patient visits, reduction of complications associated with multiple intramuscular injections, reduction of cost and administration of specific vaccines, and reduction of risk caused by delayed or missed vaccine.
In conclusion, we can say that the effectiveness of vaccines, based on the real control of the specific diseases in the population, is not affected because sometimes the antigens are used combined in a vaccine. The risk of specific diseases controlled with the vaccines selected in this study, is very low in Albania and the combined antigens have not ventured it.

A continuous downward trend of disease incidence is the most obvious evidence of optimal levels of vaccine effectiveness, and consequently the appropriate use of vaccination program. The more pronounced this trend is, the more optimal the vaccine effectiveness levels are; i.e. the more they approach to the level of that vacine effectiveness.

\section{References}

[1] Harris, J. B., Gacic-Dobo, M., Eggers, R., Brown, D. W., and Sodha, S. V. 2013. "Global Routine Vaccination Coverage.” MMWR Morb. Mortal Wkly. Rep. 63 (46): 1055-8.

[2] WHO, "Vaccines Prequalificiation Priority List", 2013-2014.

[3] WHO, “Global Routine Vaccination Coverage”, 2010.

[4] Nelaj, E. 2013. "Niveli i gjendjes imunitare ne populate per disa nga vaksinat virale dhe bakteriale" 53-104.

[5] M \& B 7 “Advantages of Combined Vaccines” (1998) 461-3.

[6] Atkinson, W., et al. 2010.

[7] Kakarriqi, E. 2002. "Epidemiological Background of Infectiuos Diseases in Albania and Their Prevention and Control in the Context of Natural Disasters and Infectiuos Diseases", 20-27, 77-81.

[8] WHO, 2010 "Immunization in Practice".

[9] Kakarriqi, E. 2003.

[10] Bino, S., et al. 2000.

[11] Aktison, W., et al. 2010; Baer, G., et al 2005.

[12] Ramsay, M. 2003; ETAG 2004.

[13] Robo, A., Simaku, A., Nelaj, E., Kakarriqi, E., and Bino, S. 2014. "Surveillance of Measles and Rubella in Albania.” International Journal of Ecosystems and Ecology Science 4 (1): 163-6.

[14] Nelaj, E., Lika, (Çekani) M., and Bino, S. 2013. "Evaluation of Albanian Immunization Program with Hepatitis B Vaccine.” Albanian J. Agric. Sci. 12 (2): 315-9.

[15] Nelaj, E., Lika, (Çekani) M., and Bino, S. 2014. "Impact of Hepatitis b Vaccination as an Important Indicator for 
Immunization Program in Albania." Journal of Life Sciences 4.

[16] Hepatitis B Vaccines: WHO position paper-reccomandations.

[17] Rubella Vaccines: WHO position paper No. 29, 86: 301-6.

[18] Pertussis Vaccines: WHO position paper No. 40, (2010),
85: 385-400.

[19] Wiley, J. 2012 "Combined DTP-HBV-HIB Vaccine versus Separately Administered DTP-HBV and HIB vaccines for Primary Prevention of Diphtheria, Tetanus, Pertussis, Hepatitis B and Haemophilus Influenzae B” (Review) Copyright $@ 2012$ The Cochrane Collaboration. 